\title{
Increasing the lateral resolution of scanning microscopes by a factor of two using 2-Image microscopy
}

\author{
Nicolas Sandeau \\ nicolas.sandeau@upmc.fr
}

\section{Laure Wawrezinieck}

\section{Patrick Ferrand}

\section{Hugues Giovannini}

\section{Hervé Rigneault}

\author{
Université Pierre et Marie Curie - Paris VI, BioMoCeTi - UMR CNRS 7033, Case Courrier 1384 Place \\ Jussieu 75252 Paris Cedex 05, France \\ CPGE Lycée Paul Valéry, Paris, France \\ Institut Fresnel, CNRS, Aix-Marseille Université, École Centrale de Marseille, Campus de Saint-Jérôme, \\ 13013 Marseille, France \\ Institut Fresnel, CNRS, Aix-Marseille Université, École Centrale de Marseille, Campus de Saint-Jérôme, \\ 13013 Marseille, France \\ Institut Fresnel, CNRS, Aix-Marseille Université, École Centrale de Marseille, Campus de Saint-Jérôme, \\ 13013 Marseille, France
}

Increasing the resolution of optical microscopes is a challenging task for studying the cell machinery at the molecular level. $4 \mathrm{Pi}$ or Total internal-reflection fluorescent microscopy (TIRF) microscopies permit one to reduce the axial dimension of the detection volume. To reduce its lateral dimension, we have proposed a solution in which the scanning head of a $4 \mathrm{Pi}$ microscope or of a confocal microscope is coupled to an interferometer. With this technique two beams coming from the source produce two images that are superimposed coherently. For this reason, one can call this technique 2-Image microscopy. It has been shown that with 2-Image microscopy, the complete use of the spatial frequencies collected by the objective allows to reach a $1.22 \lambda / 4 N A$ lateral resolution as defined by Rayleigh. This improvement is independent of the excitation mode and is effective with incoherent light such as fluorescent or chemiluminescent (i.e. without optical excitation) samples. In this paper, we present an interferometric set-up and a modulation technique that make benefit fully from the advantages of 2-Image microscopy. [DOI: 10.2971/jeos.2009.09040]

Keywords: lateral resolution, confocal microscopy, fluorescence, bioluminescence

\section{INTRODUCTION}

Studying the cell machinery at the molecular level requires ultra-sensitive, super-resolving and non invasive tools, the most common being fluorescence confocal microscopy. The $3 \mathrm{D}$ resolution of scanning microscopes is defined by a $3 \mathrm{D}$ detection efficiency function (DEF). This function is defined by the product of the excitation efficiency function (EEF) with the collection efficiency function (CEF). Each of these three functions delimits a 3D volume. To increase resolution, at least one of these volumes must be shrunk. Thus strong efforts have been made in the last decade to reduce the axial dimension (usually the largest) of the DEF volume. 4Pi [1]-[3], TIRF [4] or I5M [5] microscopes allow to reach an axial DEF dimension as small as $100 \mathrm{~nm}$. More recently other methods [6]-[10] have been developed to reduce the DEF lateral dimensions. Most of them [6]-[8] reduce the dimensions of the EEF. A solution, in which two beams coming from the incoherent point source of the sample produce two images that are superimposed coherently, has been proposed to shrink the three dimensions of the CEF [9] of 4Pi-microscopes. Since this method only acts on the collected signal, the resolution in this case is independent of the excitation mode [11]. Finally, a confocal interferometric microscope $[12,13]$ in which, as in [9], the production of two symmetrical images of the source modifies the CEF, has been proposed for improving the lateral resolution. In this paper we propose an alternative configuration for the interferometer and we discuss the possibility of modulating temporally the retardation for increasing the signal to noise ratio (SNR).

\section{PRINCIPLE OF 2-IMAGE MICROSCOPY}

With (i) 4Pi C-type [3] and (ii) 4Pi' [9] two coherent images (for each incoherent point-source) are created symmetrically with respect, (i) to the focal plane and (ii) to the focus (Figure 1) respectively. This idea applied to classical fluorescence confocal microscopes has been patented in 2006 [12]. In a 2-Image microscope (Figure 2), an interferometer generates, for each incoherent point-source of the sample, two coherent images which are symmetrical with respect to the optical axis (Figure 1) [12]. A Michelson-type interferometric set-up, where one mirror is replaced by a retroreflector corner cube has been chosen for its simplicity. With such a set-up, an emitter placed at the position $\vec{r}_{0}$ in the object focal plane creates two images in the image focal plane centered on $M \vec{r}_{0}$ and $-M \vec{r}_{0}, M$ being the magnification. The intensity recorded by the photodetector (PMT, $\mathrm{APD}$, etc.) is thus given by the function

$$
C E F_{2 I}\left(r_{0}, \varphi\right)=\frac{1}{4} \iint_{S}\left|A\left(\vec{r}-\vec{r}_{0}\right)+e^{i \varphi} A\left(\vec{r}+\vec{r}_{0}\right)\right|^{2} \mathrm{~d}^{2}(M \vec{r})
$$




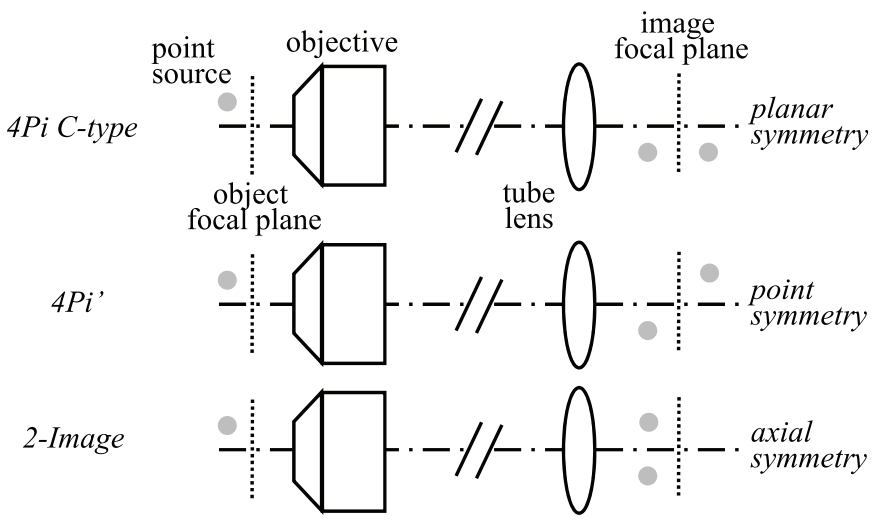

FIG. 1 Equivalent schemes of $4 \mathrm{Pi}$ C-type, $4 \mathrm{Pi}^{\prime}$ and 2 -Image microscopes. In classical confocal microscopes a point source gives one image (Airy disk). In $4 \mathrm{Pi}$ C-type microscopes a point source gives two coherent images symmetrically about the image focal plane. In $4 \mathrm{Pi}^{\prime}$ microscopes a point source gives two coherent images that are symmetric with respect to the image focus. In 2-Image microscopes a point source gives two coherent images symmetric with respect to the optical axis [12].

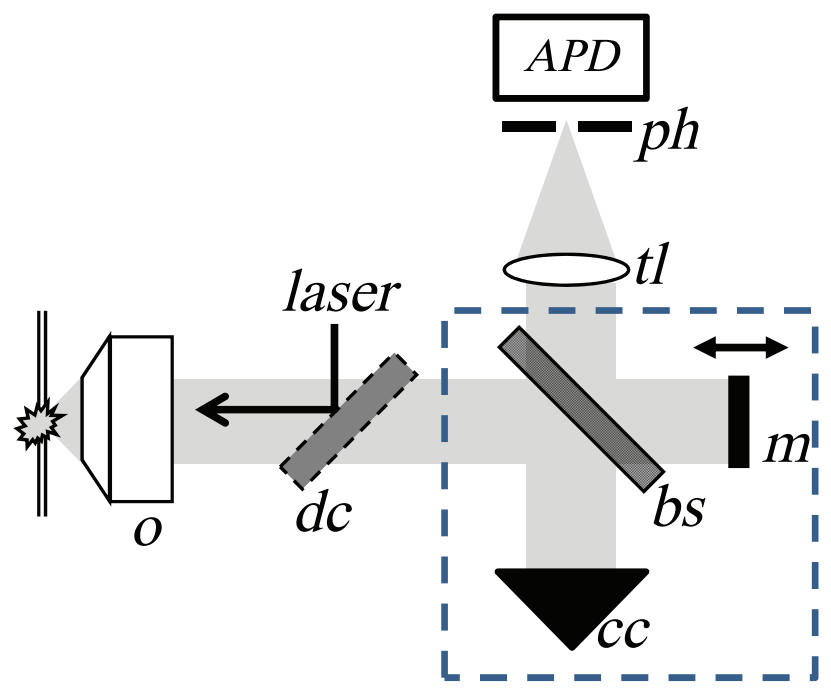

FIC. 2 Set up of a 2-Image microscope. A modified Michelson interferometer is incorporated in the tube of a classical confocal microscope. $O$ is the objective lens, $\partial c$ the dichroic mirror, bs a beam splitter, $m$ a mirror, $c c$ a retroreflector corner cube, $t$ the tube lens and $p h$ the pinhole. The metal and empty corner cube reverts one of the two images without chromaticism. This set-up can be used with all types of excitation: linear, multiphoton, with evanescent waves or chemical (i.e without optical excitation), etc.

where $A(\vec{r})$ is the scalar electric field (in the image focal plane) emitted by a point source situated in the object focal plane described by

$$
A(\vec{r})=\frac{J_{1}(k N A M\|\vec{r}\|)}{k N A M\|\vec{r}\|}
$$

$\varphi$ is the phase retardation introduced by the interferometer between the two images, $S$ the pinhole surface, $N A$ the numerical aperture of the objective and $k$ the emission wave number. In Eq. (2), $\|\vec{r}\|$ represents the $\vec{r}$ vector norm and $J_{1}$ is the Bessel function of the first kind of order 1.

Thus the $C E F_{2 I}\left(r_{0}, \varphi\right)$ function can be easily written as

$$
\operatorname{CEF}_{2 I}\left(r_{0}, \varphi\right)=\frac{1}{2}\left[\operatorname{CEF}_{\mathcal{C}}\left(r_{0}\right)+\cos (\varphi) N C E F_{2 I}\left(r_{0}\right)\right]
$$

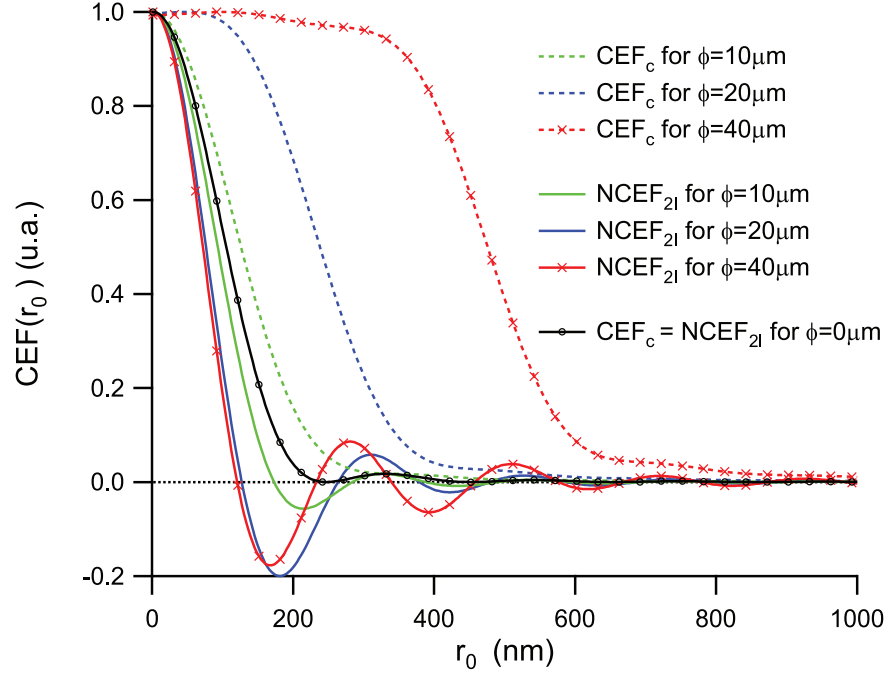

FIG. 3 Sections of normalized CEFs in the object focal plane of classical confocal and 2-Image microscopes for several pinhole diameters. The $C E F_{C}$ and $N C E F_{2 I}$ functions are respectively defined in Eqs. (4) and (5). We consider an oil ( $n=1.518)$ immersion objective with a numerical aperture $N A=1.3$, a magnification $M=40$ and a luminophore emitting at $525 \mathrm{~nm}$.

where $C E F_{c}$ is the CEF of classical confocal microscopes which is defined by

$$
C E F_{c}\left(r_{0}\right)=\iint_{S} A\left(\vec{r}-\vec{r}_{0}\right) d^{2}(M \vec{r})
$$

and

$$
\operatorname{NCEF}_{2 I}\left(r_{0}\right)=\iint_{S} A\left(\vec{r}-\vec{r}_{0}\right) A\left(\vec{r}+\vec{r}_{0}\right) d^{2}(M \vec{r}) .
$$

The $C E F_{2 I}$ function is narrower and steeper than the CEF of classical confocal microscopes as reported in [9].

In Figure 3, one can note that the $N C E F_{2 I}$ function is always narrower than $C E F_{c}$ and its width decreases when the pinhole size increases, unlike $C E F_{c}$. In fact these two functions converge towards the Airy disk function when the pinhole size tends towards zero. This characteristic can be very interesting to collect a maximum of signal with a high resolution in so far as the first term $\left(C E F_{c}\right)$ can be eliminated. Unfortunately, opening the pinhole will not only increase the signal of interest but also the background noise coming from peripheral objects, decreasing consequently the signal to noise ratio (SNR). Thus it is necessary to use a pinhole well adapted to the Airy disk as in classical confocal microscopy except in the cases where the excitation mode delimits the confocal volume as in multi-photon excitation mode.

\section{REACHING A NARROWER COLLECTION EFFICIENCY FUNCTION}

To reach $N C E F_{2 I}$, Wicker et al. suggest in [13] to use a particular Mach-Zender Interferometer (in the tube lens of the microscope) and to subtract the two complementary output intensities. This optical scheme could be very interesting to obtain resolved images even if chromaticism, aberrations of the inversion system and also instability of this type of interferometer make it difficult to implement for practical applications. Moreover the subtraction of the output intensities make 
the measurement strongly sensitive to the noise. One can note that the same result ${ }^{1}$ would be obtained by using any technique allowing the two values of $C E F_{2 I}(\varphi)$ to be subtracted i.e. $\forall \varphi_{i} \neq \varphi_{j} N C E F_{2 I} \propto C E F_{2 I}\left(\varphi_{i}\right)-C E F_{2 I}\left(\varphi_{j}\right)$.

Another solution can be given by the set-up described in Figure 2 where, thanks to a fast translation of the mirror around the zero optical path difference (OPD) between two positions corresponding to the phase retardations $\varphi_{1}$ and $\varphi_{2}$, the signal can be integrated. In this case, the integrated output signal $I\left(r_{0}\right)$ is given from Eq. (3) by

$$
I\left(r_{0}\right)=\int_{\varphi_{1}}^{\varphi_{2}} f(\varphi) C E F_{2 I}\left(r_{0}, \varphi\right) d \varphi .
$$

In order to obtain $I\left(r_{0}\right) \propto N C E F_{2 I}\left(r_{0}\right)$, the function $f(\varphi)$ has to verify the conditions

$$
\left\{\begin{array}{l}
\int_{\varphi_{1}}^{\varphi_{2}} f(\varphi) d \varphi=0 \\
\int_{\varphi_{1}}^{\varphi_{2}} f(\varphi) \cos (\varphi) d \varphi \neq 0 .
\end{array}\right.
$$

To simplify the calculation, we can choose $\varphi_{2}=-\varphi_{1}=\varphi_{0}$, with $\varphi_{0}$ small enough to keep a good interference contrast. $\varphi_{0} \leq \pi$ should be acceptable for common luminophores with a coherence length of about $5 \mu \mathrm{m}$. Several functions verifying Eq. (7) can be chosen depending on the experimental context. For example, the following choice is very simple, robust to a slight phase shift and able to work also with a discrete integration using an even number of points,

$$
f(\varphi)=\left\{\begin{array}{rll}
1 & \text { if } & |\varphi| \leq \varphi_{0} / 2 \\
-1 & \text { if } & |\varphi|>\varphi_{0} / 2
\end{array}\right.
$$

In Section 5 we develop another choice which is more accurate to minimize the noise,

$$
f(\varphi)=\cos (\varphi)
$$

With such choices the microscope described in Figure 2 has many advantages. First, a high resolution is obtained because the CEF is determined by $N C E F_{2 I}$ which is narrower than $C E F_{2 I}$. Secondly, the Michelson-type interferometer is easy to set-up, the metallic plane mirror and the corner cube enable achromaticism and introduce no geometrical aberration nor polarization modification [14]. Nevertheless, some losses of signal can be expected particularly because of the cube edges. Indeed, the corner cube must be placed in the pupil plane of the lenses in order to avoid a lateral shift of the beam.

\section{STUDY OF THE NCEF 2 FUNCTION BY MEANS OF THE OTF}

In the limit case where there is no pinhole, as reported in [13], the CEF is given by

$$
\operatorname{NCEF}_{2 I}\left(r_{0}\right)=[A \otimes A]\left(2 \vec{r}_{0}\right)
$$

with $\otimes$ being the convolution product. From Eq. (10) we obtain the optical transfer function (OTF) of collection using a 2D Fourier transform,

$$
\operatorname{OTF}_{2 I}\left(\frac{k}{k_{0}}\right)=\left[\operatorname{Circ}_{2 N A}\left(\frac{k}{k_{0}}\right)\right]^{2} \propto \operatorname{Circ}_{2 N A}\left(\frac{k}{k_{0}}\right)
$$

\footnotetext{
${ }^{1}$ In the case where the noise is not taken into account.
}

where the function $\mathrm{Circ}_{2 N A}$ is a disk with a radius equal to $2 N A$. First of all, we can conclude that in a 2-image microscope (without pinhole), all the spatial frequency information collected by the objective is fully used in the imaging process. This result can be compared to that obtained with a structured illumination in wide-field microscopy [7]. Furthermore the possible use of a pinhole would bring the major advantage leading to a strong axial resolution and to insure the enhancement of the lateral resolution (cf. Figure 5). From Eq. (11), a reverse $2 \mathrm{D}$ Fourier transform allows to rewrite $N C E F_{2 I}$ as a single Bessel function,

$$
N C E F_{2 I}\left(r_{0}\right) \propto \frac{J_{1}\left(2 k N A M\left\|\vec{r}_{0}\right\|\right)}{2 k N A M\left\|\vec{r}_{0}\right\|} .
$$

Thus the first zero is reached for $1.22 \lambda / 4 N A$ i.e. half of the well-known value $1.22 \lambda / 2 N A$ for the lateral resolution of a classical microscope, as defined with the Raylieigh criterion.

\section{CONSEQUENCES OF NOISE}

The improvement of resolution is independent of the excitation mode. Therefore one can envisage working with chemiluminescent samples [15]. However, the brightness of these kind of samples is dim and the subtraction of two output intensities (one corresponding to a constructive interference, the other corresponding to a destructive interference) worsens the SNR as shown in Figure 4. The noise in this case is 6 times stronger in images (c) than in images (a) and (b). Thus in Figures 4(a)-(d), the numerical images have been calculated for 4 types of scanning microscopies: (a) classical confocal, (b) 4Pi' [9] or 2-Image with $\varphi=0$, (c) 2-Image (configuration proposed by Wicker et al.) [13], (d) 2-Image with the modulation/demodulation technique described in this section. For these images we have taken into account a maximal detection of 1000 photons for each bioluminophore weighted by the CEF factor which depends on the respective positions of these bioluminophores. Thanks to the random noise function of Igor Pro software, we have added the resulting Poisson noise (which depends on the detected intensity) in addition to an additive electronics dark detection noise equal to 50 photons. This situation can basically tally with a bioluminescence detection. One can note that the two parts of the object will be discriminated at different positions according to the technique of microscopy used. These results are shown in Figures 4(e)(h). The resolution (and partly the noise) allows one to distinguish objects separated by (e) $475 \mathrm{~nm}$ for a classical confocal microscope, (f) $283 \mathrm{~nm}$ for $4 \mathrm{Pi}^{\prime}$ microscope (or equivalent) and (g) and (h) are from $222 \mathrm{~nm}$ for 2-Image microscope according to the level of noise. As a result, in order to obtain both high resolution and strong SNR, 2-Image microscopy requires to use an appropriate modulation/demodulation technique.

The noise can be taken into account in Eq. (3) by making $C E F_{C}$ and NCEF dependent on time. To suppress the noise, we can record the time depending signal for each point of the scan $C E F_{2 I}\left(r_{0}, t\right)$ with a simple phase ramp of amplitude of $2 \pi$ centered on $0\left(\forall t \in\left[\frac{n}{f_{0}}, \frac{n+1}{f_{0}}\right]\right.$ with $n \in \mathbb{N}$, $\left.\varphi(t)=2 \pi f_{0} t-(2 n+1) \pi\right)$. With this choice, Eq. (3) can be 

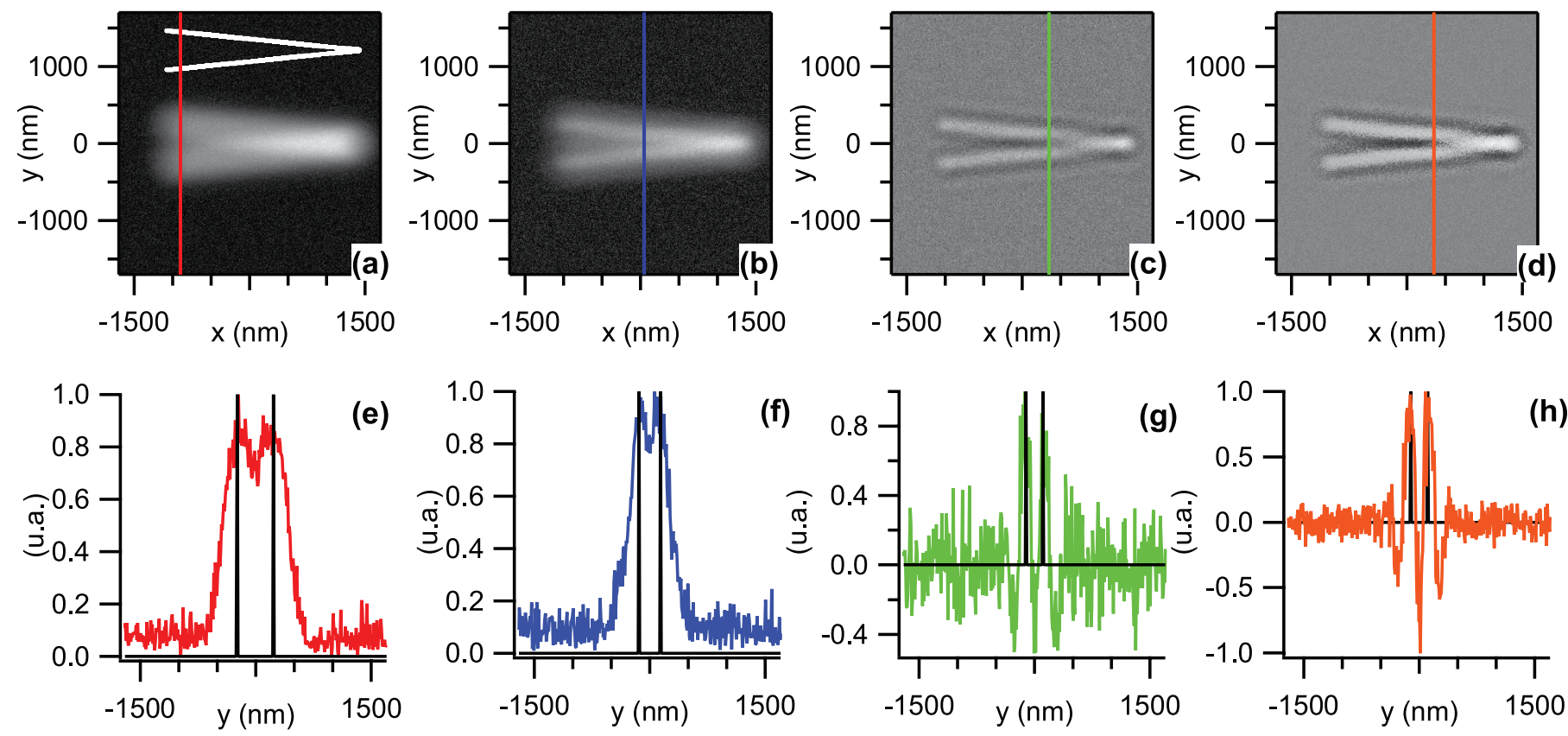

FIG. 4 (a)-(d): Numerical images with noise of a bioluminescent "V" object situated in the focal plane of the objective and depicted in the top of image (a). (e)-(h) represent respectively a normalized section of images (a)-(d) represented by the colored line on the respective image. The positions of the lines are chosen such as the discrimination limit of the two parts of the object (depicted in black in each graph (e)-(h)) is reached. (a) and (e) are obtained with a classical confocal microscope, (b) and (f) with a 4 Pi' microscope [9], (c) and (g) with the 2-Image microscope proposed by Wicker et al. [13] and, (d) and (h) with the 2-Image microscope proposed in this paper with the sinusoidal modulation/demodulation technique described in Section 5 . The characteristics of these microscopes are: $N A=1.3$ in oil, $M=40$ and the pinhole diameter $\phi=20 \mu \mathrm{m}$. We consider a chemi-luminophore emitting at $525 \mathrm{~nm}$.

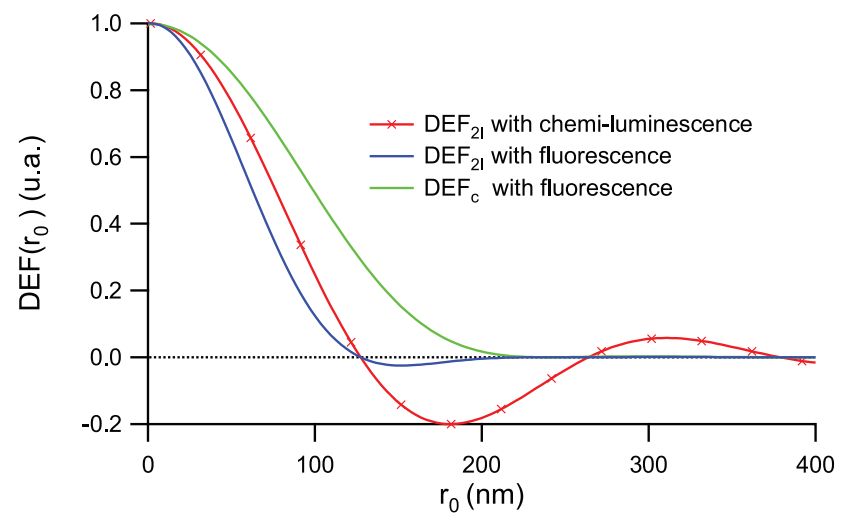

FIG. 5 Sections of normalized DEF in the object focal plane of classical confocal ( $\left.D E F_{c}\right)$ and 2-Image microscopes $\left(D E F_{2 I}\right)$. We consider a fluorophore (excited at $488 \mathrm{~nm}$ ) and a chemi-luminophore both emitting at $525 \mathrm{~nm}$. $N A=1.3$ in oil, $M=40$ and the pinhole diameter $\phi=20 \mu \mathrm{m}$.

written as

$$
C E F_{2 I}\left(r_{0}, t\right)=\frac{1}{2}\left[C E F_{C}\left(r_{0}, t\right)-\cos \left(2 \pi f_{0} t\right) N C E F_{2 I}\left(r_{0}, t\right)\right]
$$

We can extract two kinds of information from the discrete Fourier transform of Eq. (13); the first term $\left(C E F_{c}\right)$ corresponds to a high energy peak centered on the frequency 0 whereas the second term $\left(N C E F_{2 I}\right)$ which contains the most interesting information corresponds to a lower energy peak centered around the vibration frequency $f_{0}$ of the Michelson mirror. By filtering with a bandpass filter centered around frequency $f_{0}$, we can suppress the first term $C E F_{c}$, nearly all the dark electronic noise (additive noise) but only a part of the Poisson noise. Since the frequency filtering of the signal obtained with a linear modulation will decrease the signal a lot more than it is effective against the noise, the SNR is worsened. The same problem is met by all the techniques which attempt to improve the resolution only by suppressing the first term, such as the subtraction method described by Wicker et al. [13] as shown on Figure 4(g). One can note that this frequency filtering method is equivalent to the synchronous detection technique proposed in Section 3, with $\varphi_{0}=\pi, f(\varphi)=\cos (\varphi)$ in Eq. (6).

To eliminate more of the Poisson noise and consequently maximize the SNR, we need to use a more sophisticated numerical treatment. As it is commonly done in electronics for modulation/demodulation purposes [16], we have applied a sinusoidal modulation of the phase retardation of the Michelson (i.e. $\varphi(t)=\pi \cos (\omega t)$ ) and used an appropriate numerical treatment to reduce the noise by a factor higher than 4 , as shown in Figures 4(d) and 4(h). This demodulation treatment consists in filtering the Bessel harmonics coming from the Fourier transform of Eq. (3) where $\varphi(t)=\pi \cos (\omega t)$. This treatment corresponds in fact to the synchronous detection technique which is proposed in Section 3, with $\varphi_{0}=\pi$, $f(\varphi)=\cos (\varphi)$ in Eq. (6). After a change of variable ( $\varphi$ into $t$ ) we obtain for each point of the image with the following intensity,

$$
I\left(r_{0}\right)=\omega \pi \int_{0}^{\frac{\pi}{\omega}} C E F_{2 I}\left(r_{0}, t\right) \cos (\pi \cos (\omega t)) \sin (\omega t) d t .
$$

However, some more effective techniques of demodulation developed in electronics and adapted to the experiments should allow one to reach higher levels of SNR so these tech- 
niques should be the solution to obtain both resolved and contrasted images.

\section{APPLICATION TO 3D SAMPLES}

In 3D samples a pinhole is needed in the case of linear excitation; in particular, in order to obtain an acceptable axial resolution and to reduce the potential noise coming from out-focused planes of the sample. Figure 3 shows that a pinhole with the size of the Airy disk allows to reach approximately the maximum of the lateral resolution. Since the 2Image interferometer does not affect the axial resolution of the microscope, this size of pinhole should be a good compromise in maximize both $3 \mathrm{D}$ resolution and the signal to noise ratio. For the calculations (Figures 3 and 5), we have considered an oil ( $n=1.518)$ immersion microscope objective with an effective numerical aperture $N A=1.3$, a magnification $M=40$ and luminophores (fluorophores or chemiluminophores) emitting at $525 \mathrm{~nm}$. In these conditions, the best solution to get a high $3 \mathrm{D}$ resolution with a maximum of signal, is to use a pinhole with a $20 \mu \mathrm{m}$ diameter (the size of the Airy disk). Fluorophores are usually brighter than chemiluminophores. However, the major difference between fluorophores and chemi-luminophores ${ }^{2}$ relies on the excitation mode: fluorophores need optical excitation whereas chemiluminophores are chemically excited. Thus the detection efficiency function of the 2-Image microscope $\left(D E F_{2 I}\right)$ is defined in the case of fluorescent samples by $D E F_{2 I}=N C E F_{2 I} \times E E F$ where $E E F$ is the excitation efficiency function. On the other hand, in the case of chemi-luminescence the detection function is strictly identical to the collection efficiency function defined in Eq. (5) (i.e. $D E F_{2 I}=N C E F_{2 I}$ ). In Figure 5, $E E F$ has been calculated with the scalar theory of diffraction first proposed by Debye [17].

Several criteria can be used to evaluate the resolution of a microscope. We have decided to study two of them; either the full width at half maximum (FWHM) of the detection efficiency functions $(D E F)$ or the position of its first zero. The first zero position is ruled by the EEF in a classical confocal microscope, whereas it is driven by the collection function $\left(N C E F_{2 I}\right)$ in a 2-Image microscope: as seen in Figure 5, the first zero is the same with fluorophores (with optical excitation) and with chemi-luminophores (without optical excitation). With the set up of Figure 2) and an excitation wavelength at $488 \mathrm{~nm}$, the 2-Image microscope enables to get a first zero at $r_{0}=125 \mathrm{~nm}$, which has to be compared to $r_{0}=239 \mathrm{~nm}$ for a classical confocal microscope. For the FWHM, the EEF has a significant effect in both microscopes. With fluorescent samples, the FWHM is 1.6 times smaller in the case of the 2-Image microscope corresponding to a classical microscope with an effective numerical aperture $N A=2.15$.

In the specific case of multiphoton excitation fluorescence mode (in samples that are not too diffusing), no pinhole is needed [18] and thus the $D E F_{2 I}$ and consequently the reso-

\footnotetext{
${ }^{2}$ One can note that bio-luminophores are chemi-luminophores naturally present in biological organisms.
}

lution are delimited by the $E E F^{3}$ in the axial direction and by $N C E F_{2 I}$ in lateral dimensions.

\section{CONCLUSION}

In summary, 2-Image microscopy reduces strongly the lateral dimension of the collection efficiency volume reducing in the same amount the lateral extension of the detection efficiency volume. Since this technique acts only on the collection efficiency, it is well suited to any excitation modes: linear, multiphoton and even without optical excitation as in the particular case of chemi-luminescence. 2-image microscopy can be used to image non-luminescent samples lighted by a spatially incoherent source. Moreover, 2-Image microscopy can be adapted to all types of incoherent scanning microscopes. Indeed, the 2-Image microscope can be coupled to TIRF or to 4Pi A-type [12] microscopes, thus enabling a high 3D resolution $\left(125 \times 125 \times 100 \mathrm{~nm}^{3}\right)$. In this last case, the $3 \mathrm{D}$ resolution is the same as that obtained with the 4Pi' microscope [9], without the instability characterizing a $4 \mathrm{Pi} \mathrm{C}$-type microscope and with no aberration coming from the image inversion system.

The 2-Image microscope should be useful for applications which need an observation volume smaller than the classical limits imposed by objective microscopes. For example, Wawrezinieck et al. have shown [19] that fluorescence correlation spectroscopy (FCS) measurements at different spatial scales enable to distinguish between different submicron confinement models. Thus a 2-Image microscope used as a FCS set-up allows one to reach another range of detection volumes (see Figure 3) revealing probably the ultrafine cell membrane organization with more flexibility than using the method described in [8].

\section{ACKNOWLED GEMENTS}

The authors thank M. Pianta for her useful contribution.

\section{References}

[1] S. Hell, "Double-confocal scanning microscope" European Patent EP0491289 (1992).

[2] C. J. R. Sheppard, and Y. R. Gong, "Improvement in axial resolution by interference confocal microscopy" Optik 87, 129-132 (1991).

[3] S. Hell, and E. H. K. Stelzer, "Properties of a 4pi confocal fluorescence microscope" J. Opt. Soc. Am. A 9, 2159-2166 (1992).

[4] D. Axelrod, N. L. Thomson, and T. P. Burghardt, "Total internalreflection fluorescent microscopy" J. Microsc-0xford 129, 19-28 (1983).

[5] M. G. L. Gustafsson, D. A. Agard, and J. W. Sedat, "I5M: 3D widefield light microscopy with better than 10onm axial resolution" J. Microsc-0xford 195, 10-16 (1999).

[6] S. W. Hell, and J. Wichmann, "Breaking the diffraction resolution limit by stimulated emission" Opt. Lett. 19, 780-782 (1994).

${ }^{3}$ In the case n-photon excitation mode, the excitation efficiency function is defined by $E E F=I^{n}$ where $I$ is the intensity of the focused excitation beam. 
[7] M. G. L. Gustafsson, "Surpassing the lateral resolution limit by a factor of two using structured illumination microscopy" J. Microsc0xford 198, 82-87 (2000).

[8] J. Wenger, F. Conchonaud, J. Dintinger, L. Wawrezinieck, T. W. Ebbesen, H. Rigneault, D. Marguet, and P.-F. Lenne, "Diffusion analysis within single nanometric apertures reveals the ultrafine cell membrane organization" Biophys. J. 92, 913-919 (2007).

[9] N. Sandeau, and H. Giovannini, "Increasing the lateral resolution of 4 pi fluorescence microscopes" J. Opt. Soc. Am. A 23, 1089-1095 (2006).

[10] D. K. Kang, and D. G. Gweon, "Enhancement of lateral resolution in confocal self-interference microscopy" Opt. Lett. 28, 2470-2472 (2003).

[11] N. Sandeau, and H. Giovannini, "Arrangement of a $4 \mathrm{Pi}$ microscope for reducing the confocal detection volume with two-photon excitation" Opt. Commun. 264, 123-129 (2006).

[12] N. Sandeau, H. Giovannini, and H. Rigneault, "Interferometric confocal microscope" World Patent W0/2007/141409 (2007).

[13] K. Wicker, and R. Heintzmann, “Interferometric resolution im- provement for confocal microscopes" Opt. Express 15, 12206-12216 (2007).

[14] J. Liu, and R. M. A. Azzam, "Polarization properties of corner-cube retroreflectors: theory and experiment" Appl. Optics 36, 1553-1559 (1997).

[15] R. Creton, and L. F. Jaffe, "Chemiluminescence microscopy as a tool in biomedical research" Biotechniques 31, 1098-1105 (2001)

[16] R. E. Ziemer, and W. H. Tranter, Principles of Communications (Wiley Publishing, 2008).

[17] P. Debye, "Das verhalten von lichtwellen in der nahe eines brennpunktes oder einer brennlinie" Ann. Phys. 335, 755-776 (1909).

[18] C. J. R. Sheppard, and M. Gu, "Image formation in two-photon fluorescence microscopy" Optik 86, 104-106 (1990).

[19] L. Wawrezinieck, H. Rigneault, D. Marguet, and P.-F. Lenne, “Fluorescence correlation spectroscopy diffusion laws to probe the submicron cell membrane organization" Biophys. J. 89, 4029-4042 (2005). 